\title{
Reflection on Professional Qualification and Training for Talents of Internationalization of Traditional Chinese Medicine
}

\author{
Yang $\mathrm{Xia}^{1, \mathrm{a}}$, Wang Haidong ${ }^{2}$, Chen $\mathrm{Ji}^{1,2, \mathrm{~b} *}$ \\ ${ }^{1}$ Chengdu University of Traditional Chinese Medicine, Chengdu, Sichuan, China \\ ${ }^{2}$ International Chinese Medicine Brand Committee, World Federation of Chinese Medicine Societies, Beijing, China \\ atyryang@126.com \\ b*jeanelle_chin@126.com
}

\begin{abstract}
In order to better adapt to the internationalization of traditional Chinese medicine (TCM), the cultivation of internationalized talents of TCM needs to be strengthened. Internationalized talents of TCM should possess profound cultural foundation of TCM, exquisite professional skills of TCM, fluent foreign language expression ability, clear international idea and awareness, and strong cross-cultural communication ability. However, there are many problems in TCM international talents training at present, such as lack of comprehensive talents, clear training objectives, complete curriculum system, systematic practice, and perfect teaching staff construction, etc. These problems are the main obstacles of the development of TCM international talents strategy. This paper attempts to explore the cultivation path of TCM international talents from the aspects of strengthening international awareness, clarifying clear training objectives, optimizing the curriculum system, strengthening the professional skills training and the construction of teachers, to help the overseas transmission and development of TCM culture.
\end{abstract}

Keywords: traditional Chinese medicine, culture, internationalization, talent cultivation

\section{中医药国际化人才的专业素养与培养路径思考}

\section{杨霞 $1, \mathrm{a}$, 王海东 ${ }^{2}$, 陈骥 $1,2, \mathrm{~b}$ *}

\author{
${ }^{1}$ 成都中医药大学基础医学院, 成都, 四川, 中国 \\ 2 世界中医药学会联合会中医药国际化品牌专委会, 北京, 中国 \\ atyryang@126.com \\ b*jeanelle_chin@126.com
}

\section{摘要}

为更好地适应中医药的国际化进程, 中医药国际化人才的培养亟待加强。中医药国际化人才应该具备深厚的中 医药文化涵养、精湛的中医专业技能、流利的外语表达能力、清晰的国际理念和国际意识和较强的跨文化传播 能力。但现阶段的中医药国际人才培养工作存在很多问题，例如综合性人才少、培养目标不明确、缺乏完整的 课程体系、缺乏系统性的实践环节，以及师资队伍建设不够完善等，这些问题是阻碍中医药国际化人才战略发 展的主要因素。本文试从增强国际意识、明确人才培养目标, 优化课程体系, 加强专业技能培养、加强国际化 师资队伍建设等方面探索中医药国际人才的培养路径, 以期助力于中医药文化的海外传播与发展。

关键词: 中医药; 文化; 国际化; 人才培养 


\section{1.引言}

两千多年以来，中医药文化是中国优秀传统文化 的精髓, 一直守护着中华民族的健康。随着全球化的 推进, 中医药文化也传播到了海外国家并逐步得到认 可。中医药对外交流的深度和广度不断加大, 中医药 人才的国际化要求也相应提高。自新冠病毒疫情暴发 以来, 中医药在抗击疫情过程中发挥了重要作用, 取 得显著临床疗效, 引起世界的广泛关注。这是中医药 向世界传播的重要机遇, 而当前中医药的国际化人才 短缺或层次不高是中医药对外传播中的重要的挑战。 因此, 如何培养更多高素质中医药国际人才是当前亟 待解决的问题。本文试从中医药国际化人才的专业素 养和培养路径等方面进行探讨。

\section{2.中医药国际人才需要的专业素养}

\section{1 深厚的中医药文化涵养}

文化是一个民族的灵魂。中华民族在几千年历史 中创造和延续的中华优秀传统文化, 是中华民族的根 和魂 ${ }^{[1]}$ 。中医药文化是一种在中国传统文化思想指导 下形成的科学文化, 是中华民族几千年来的智慧的结 晶。中医文化研究涉及多学科多领域, 其提倡和遵循 的核心观点主要包含“天人合一、大医精诚、医乃仁术、 治未病”等, 顺应整体观的指导 ${ }^{[2]}$ 。中医药文化来自中 国古代儒家、道家和佛家等传统文化, 融入了人文精 神, 带有明显的中国特色, 也包含了很多哲学思想, 所以在理解时有一定难度。但因为文化是中医药内容 的核心, 对文化的理解影响着中医药的对外传播, 只 有深入理解与中医药相关的文化内涵, 才能向世界人 民展示中医药的文化渊源; 否则在中医药文化外宣时 会因为传播者理解不正确而造成文化误读与误传, 影 响中医药的国际传播, 阻碍中医药事业的发展。

\section{2 精湛的中医专业技能}

实践是检验真理的唯一标准, 中医学是一门实践 性极强的科学, 中医药理论的内涵, 只有在临床实践 中才能深刻领悟。中医药有着独特的实操技能, 包括 最基本的望、闻、问、切四诊方法、舌诊、脉诊等诊 断技能, 以及临床用药、针炎、推拿等运用技能。中 医药人才要做到能够熟练运用专业技能为患者诊疗, 并口头讲解这些技能的主要含义和作用等。作为中医 药的国际人才, 具有较高的中医专业技能是必不可少 的。只有娴熟掌握各种相关的技能, 才能在国际交流 中充分展现中医药真实的特点及疗效, 提高国外人民 对中医药的信任感。

\section{3 流利的外语表达能力}

语言是沟通交流的桥梁，在中医药对外传播中外 语是一个特别重要的部分。中医药国际人才是中医药 国际传播的主要力量, 他们不仅要能准确理解中医药
文化的内涵, 还要具有较高的外语听、说、读、写能 力, 专业外语能力能够满足对外学术交流、阅读外文 专业文献、撰写外文学术论文的需要。具体的看, 主 要包含两个方面: 在临床上, 能做到用外语特别是英 语与外国患者恰当地交流，完成接诊、治疗等环节; 在学术上, 能用外语在口头上、书面上宣传中医药知 识和文化, 阐述中医药思想, 表达学术观点。中医药 国际人才的外语水平满足这些要求, 就能顺畅准确地 向外传达中医药文化的内容, 提高世界人民对中医药 的了解和认知, 助力于中医药走向世界。

\section{4 清晰的国际理念和国际意识}

随着经济全球化，人们的国际理念和国际意识逐 渐加强。我国的中医药文化也逐步传播到其他国家, 不再是一国的专利, 可以造福全人类。中医药国际人 才必须要树立国际理念, 了解当今世界发展趋势, 并 对传播国的基本国情、政体文化、民俗风情等有一定 的认识, 才能更好地将中医药文化推向海外国家，提 高国外人民对中医药的认可, 切不可故步自封, 夜郎 自大。同时也要树立国际合作理念，积极与国外相关 机构联系和合作，通过国际间的合作推广中医药，提 高世界人民对中医药的认可, 真正助力于中医药的海 外传播。

\section{5 较强的跨文化传播能力}

跨文化传播是指来自不同文化体系的个人及组 织、国家等社会群体之间进行的信息传播活动 ${ }^{[3]}$ 。它 涉及不同的民族、不同的国家以及不同的文化背景， 且渗透在人类社会的一切交流活动中。中医药有着科 学的理论体系、丰富的临床经验和系统的医学典籍, 是无数先辈在长期的实践中探索出的治病救人和医 疗保健的诊疗技术和方药体系, 是几千年医学理论和 实践积累的结晶。中医药的国际人才必须要有较强的 跨文化传播能力, 第一, 要能够理解中医药文化的深 刻内涵和其他国家的一些背景文化，在文化交流中就 能找到有针对性的传播方式，促进中医药的海外传播。 第二, 要能够熟练使用外语, 语言是沟通交流最直接 的载体和文化融入的最便捷的桥梁。第三, 在跨文化 传播时运用合适的视角和途径, 选准目标和切入点, 让中医药文化深入人心。

\section{3.中医药国际人才培养现阶段的问题}

\section{1 综合性人才少, 培养层次不高}

近年来, 国家逐步认识到中医药的重要性, 加大 了中医药的人才培养, 但总体上培养的国际人才数量 与中医药国际合作和交流需要的数量仍有较大差距。 当前中医药国际人才的培养存在着两个主要问题, 第 一, 培训水平低, 第二, 人才数量较少。这些都是阻 碍中医药文化国际交流的问题。有专家指出, 中国人 才资源总量很大, 但高层次人才只占人才资源总量的 
$5.5 \%$ 左右，而高级人才中的国际化人才更少。当前我 国的中医药国际人才培养处于“杂而不多，杂而不精” 的状态，一些人才懂外语却不懂看病，一些人才懂看 病的又不精通外语, 不利于中医药的海外传播。总的 来说, 虽然目前我国的中医药国际人才数量在增加, 但总体上还是处于缺乏状态, 中医药人才的综合素质 也有待提高。

\section{2 缺乏清晰的培养目标}

目前国家对中医药的国际化人才没有出台相关 教育指导方案确定培养目标和要求, 受地域、经济、 教育等的影响, 各地高校对国际化人才的理解也各有 差异, 培养缺少统一的标准和要求。中医药是我国土 生土长的传统医学, 课程内容和人才培养模式较为独 特, 很多业内人士形成了“只有在中国才能学习中医、 学习中医不需要走出去”的刻板映像, 中医药国际化 动机不足。各个高校对人才的培养也大多局限于满足 国内需求, 对国际化人才重视度不够, 对中医药文化 宣传、中医药标准等方面的人才培养少, 导致高校的 学科建设和学生培养目标制定都缺乏国际化导向, 这 是造成国际人才不足的重要的原因之一。培养目标缺 失, 不知道该培养什么样的中医药人才, 成为阻碍中 医药国际化事业发展的重要因素。

\section{3 缺乏完整的课程体系}

课程体系的问题主要体现在以下几个方面: 第一, 中医基础薄弱, 中医专业学生中西医课程分布不合理。 据调查, 中医专业的本科生被安排了大量的西医课程, 西医课程占的比例逐渐升高, 中医传统文化的课程越 来越少, 中医药学与传统文化的有机联系未能很好体 现, 学生可能缺乏中医药文化知识, 不能很好向外传 播。第二, 课程设置与结构相对单一, 语言类课程专 业化程度底。各大中医高校中医专业的学生开设英语 课时少, 同时一些有语言专业的高校开设的中医药类 课程少, 跨文化类课程几乎没有, 这导致中医药就是 中医药, 语言就是语言, 二者不可以贯通, 严重阻碍 中医药的国际交流。第三, 各中医院校在具体的教育 教学环节中没有充分考虑留学生的专业需求, 文化背 景、宗教信仰, 语言能力以及学习能力, 没有合理的 规划调整课程设置、教学模式、教材选择等 ${ }^{[4]}$ 。在课 程编排上中国传统文化、古汉语等课程少, 使留学生 难以理解一些知识, 学习难度大; 临床实习方面留学 生的操作也比较少。这些问题都是中医药国际人才培 养的制约因素。

\section{4 缺乏系统性的实践环节}

各中医院校是中医药人才培养的主要基地, 但长 期以来中医院校学生的教育重知识传授, 轻能力培养, 学生的主要精力和时间用于听说些、啃书本、背概念, 通过机械地记忆去消化前人在临床实践中总结的经 验, 在教学上属于“灌输式、填鸭式”。医学是一门实
践性很强的学科, 只学习理论知识是远远不够的, 临 床技能是其中十分重要的部分。现在各中医院校中医 专业的本科学生学制为 5 年, 但前四年都是以理论知 识学习为主, 几乎没有实践环节, 学生也没有机会进 行临床实践操作; 到第五年会安排医院实践实习, 但 这种实践模式不成系统性, 可能会造成学习的理论知 识不能立即得到实践论证而掌握不好, 不能真正让学 生掌握相关专业技能。缺乏系统性的实践, 专业技能 宽乏, 是阻碍中医药国际人才的培养工作的重要制约 因素。

\section{5 师资队伍建设不够完善}

师资是中医药人才培养的关键，提高教师的整体 素质是必不可少的。当前师资队伍存在很多问题: 第 一, 一部分中医药专业具有过硬的中医专业知识和较 高的临床诊疗技能，但外语语言能力相对薄弱，无法 胜任双语教学; 一些高校为此引进了有海外留学经历 和专业外语院校毕业的教师，但这些教师大多没有中 医药学专业背景, 无法准确详实的传递知识信息。第 二, 很多临床带教教师有着丰富的临床诊疗经验, 但 在医院病人多、环境比较杂乱, 教师在临床教学中没 有足够的时间与实习学生进行沟通交流, 学生没有机 会学习一些经典病例, 无法精进专业知识。第三, 近 年来中医药留学生不断增加, 但教师缺少对国外文化 背景及知识背景的了解, 不能和留学生很好的沟通。 因此, 缺乏国际化、一体化教师队伍成为了高校培养 中医药国际人才的重要制约因素。

\section{4.中医药国际人才的培养路径探索}

\section{1 增强国际化意识, 培养创新型综合人才和 优秀留学生}

首先, 在培养国际人才时要加强国际意识的培养, 了解当今世界的发展趋势, 紧跟时代步伐培养中医药 国际人才才能真正将中医药传向世界。努力培养中医 药专业知识扎实, 熟练使用外国语言, 熟悉国际规则 的中医药教育、中医药翻译、中医药文化交流、中医 药服务贸易等综合性人才。同时，要重视中医药国际 人才的跨文化传播能力培养, 打造全面发展的中医药 传播人才。

在中医药走向世界的征程中, 国外来华的中医药 留学生也是重要的一环。要培养精通专业知识, 临床 技能过硬的留学生，在他们回国后向他们本国的人民 展示中医药, 促进中医药的传播。如果留学生在华学 习没有对中医药有一个准确的认识, 很可能会误导一 些人对中医药的认识。同时, 加快中医药留学生的流 动性 ${ }^{[5]}$, 在接受国外来华留学生的同时也要增加我国 外派留学生的数量, 促进中医药的国际交流, 助力于 中医药走向世界。 


\section{2 明确中医药国际人才培养目标, 健全保障 制度}

确立中医药国际人才的培养目标是做好人才培 养工作的首要条件, 有目标才会有针对性的培养方案。 加强中医药国际人才的培养也是推动世界中医药发 展的需要, 所以要努力培养具有全球视野, 较强的国 际竞争力创造力, 具有跨文化交流能力、具有先进的 国际合作意识以及国际化理念, 熟悉掌握国际医学事 务规则管理、过硬的外语语言能力, 并精通掌握中医 药专业知识的高级专业人才。培养有较高跨文化传播 能力的中医药国际人才, 促进中医药的对外交流与传 播。高校可以根据自身特点及办学自主权积极探索有 利于中医药人才培养的路径, 比如考虑和一些与中医 药相关的行业合作培养。

中医药国际人才的培养, 需要国家政府机关, 企 业单位等出台相关的政策和法律法规, 为其提供有力 的支持和保障。高校也可通过设立申请中国政府奖学 金、自主设立奖学金、助学金、科研基金等鼓励中医 药学生投身中医药的国际化事业, 为我国的中医药高 等教育与国际接轨提供制度保障。

\section{3 优化课程结构, 构建中医药国际化课程体 系}

要促进中医药国际人才的培养, 进一步提升人才 的创新能力, 各中医院校就必须要优化和改革现有的 课程结构, 使之与国际接轨, 培养具有较强跨文化传 播能力的中医药国际人才和留学生。第一, 要合理安 排中医和西医课程, 二者所占的比例要合理, 开设足 够的中医专业知识和传统文化的课程, 这样才能使中 医药传统文化得到传承, 也帮助学生更好的理解中医 知识。第二, 要重视专业英语和跨文化课程的设置, 将英语和专业知识的学习课程有效结合起来, 提高学 生的语言应用能力。不仅以考试结果考核学生, 重视 语言的运用, 鼓励学生学以致用, 尝试用外语接诊或 做学生交流, 有机会还可以走出国门在外语的环境中 努力提升中医药专业知识。同时也可与国外高校合作, 开发线上课程, 引进海外优质资源, 在学习中医药的 同时也多了解现代医学并了解专业的外语表达, 增强 辩证思维和独立思考能力, 从而培养“学贯中西”的复 合型人才。第三, 各高校在编排课程时要充分考虑留 学生的实际情况, 可以多开设与中医有关的中国传统 文化课程, 帮助留学生更好更深入的了解中医文化。 增加临床实践机会, 让留学生充分掌握中医临床技能, 塑造中医药国际传播的重要力量。

\section{4 加强中医专业技能培养, 增加临床实践}

中医的临床技能与临床思维需要在临床实践中 不断打磨与提升。加强学生的中医专业技能是培养中 医药国际化人才的重要举措, 可以通过如下的几个方 面提高学生的临床实践技能: 第一, 完善现有的中医
实践教学制度。现在五年学制的中医专业学生只有在 最后一年才会去医院临床实践, 这种制度可能导致前 几年学的理论知识不能很好掌握, 因为理论教学和实 践相隔时间太久。应该完善这种教学制度, 让理论教 学和临床教学充分结合, 增加临床实践。第二, 加大 中医院校临床基地的内涵建设。以学科建设为龙头, 带动中医药院校临床实践基地建设, 形成规范化的临 床教学管理体系, 提高临床教学质量, 培养专业技能 过关的国际人才。

\section{5 加强师资队伍建设，保障师资国际化水平}

目前, 各高等中医药院校也迫切需要培养一批具 有丰富理论课教学经验、临床带教经验、熟练的外语 语言交际能力以及良好职业素养的一体化教师队伍。 第一, 要培养既精通中医药专业知识, 又拥有外语应 用技能的教师，使之成为中医药国际人才培养的重要 成员。不断提升教师的教学能力、职业素养, 鼓励一 些教师参加行业交流继续深造，提供一些出国交流的 机会，提高教师队伍的综合素质。第二，增加临床教 学老师数量, 给学生深入讲解典型病例, 以提高学生 的专业技能, 培养合格的专业人才。第三, 培养具有 国际视野的教师队伍, 了解留学生的实际情况, 包括 文化背景、语言等, 因材施教, 培养中医专业知识扎 实、临床技能合格的留学生, 使之成为中医药的优秀 名片。

\section{5.结论}

中医药的国际发展离不开人才的培养, 高水平的 国际人才对中医药的现代化和国际化具有重要的意 义。中医药国际人才的培养是一个长期的、复杂的、 系统的工程, 现在正处于不断探索的阶段, 在这个过 程中会遇到一些挑战, 要努力解决遇到的问题, 根据 国际需求培养相应的国际人才。充分考虑各方面的因 素，努力培养高层次的、跨文化传播能力强的综合性 国际人才, 助力于中医药的海外传播, 促进中医药事 业的发展。

\section{项目基金}

本文为国家社科基金西部项目《中医药非物质文 化遗产在澳大利亚的跨文化传播研究》(19XXW005) 的阶段性成果之一。

\section{REFERENCES}

[1] Liu Y, Cheng WD, Yuan LX, et al. (2020) Confidence in Culture and Inheritance and Development of Traditional Chinese Medicine --Consideration and Exploration of Ideological and Political Education in the Course of Traditional Chinese Medicine. Chinese Medicine Modern Distance Education of China, 21: 27-29. 
[2] Shang B, Ju BS, Liu ZL, et al. (2018) How to Guide the Cross Cultural Communication of Chinese Medicine with the Core Values of TCM Culture. Journal of Basic Chinese Medicine, 6: 775-777.

[3] Liu GW. (2011) On intercultural communication of TCM. China Journal of Traditional Chinese Medicine and Pharmacy, 5: 1047-1050.

[4] Li X. (2017) Analysis on the Current Situation and Strategy of International Talent Cultivation of Traditional Chinese Medicine. China Continuing Medical Education, 8: 52-54.

[5] Pan M, Li XM, Ying SL. (2016) Research on the Countermeasures of Accelerating the Cultivation of Innovative Talents in the International Education of Chinese Medicine. Guangming Journal of Chinese Medicine, 17: 2588-2590. 